Original Research Paper

\title{
A Blockchain-based Renewable Energy Investment Management Platform: Decentralized Sustainable Development (DeSDev)
}

\author{
${ }^{1}$ Warodom Werapun, ${ }^{2,3}$ Tanwa Arpornthip, ${ }^{1}$ Esther Sangiamkul, \\ ${ }^{1}$ Rattana Wetprasit and ${ }^{1}$ Tanakorn Karode \\ ${ }^{1}$ College of Computing, Prince of Songkla University, Phuket, Thailand \\ ${ }^{2}$ Faculty of Technology and Environment, Prince of Songkla University, Phuket, Thailand \\ ${ }^{3}$ Andaman Environment and Natural Disaster Research Center, Prince of Songkla University, Phuket, Thailand
}

\author{
Article history \\ Received: 09-10-2020 \\ Revised: 25-11-2020 \\ Accepted: 26-11-2020 \\ Corresponding Author: \\ Warodom Werapun \\ College of Computing, Prince \\ of Songkla University, Phuket, \\ Thailand \\ Email: warodom.w@psu.ac.th
}

\begin{abstract}
In this study, a novel blockchain-based platform for renewable energy investment is proposed. The blockchain technology has been shown to be a reliable way to send financial transactions across the world in a decentralized manner, at a fraction of the cost and with a faster transaction time. The proposed platform is capable of handling an equity-sharing investment program for solar PV projects. The platform distributes the electricity generation income by sending it over a blockchain. The Ethereum blockchain is used to create a prototype, which is a smart contract. The prototype is tested with a solar-PV electricity generation data. The result shows that the proposed platform can handle the expected transactions without an error. The overall performance of the proposed platform is tested by comparing its temporal duration and its financial costs with centralized solutions. Therefore, the proposed platform provides all the benefits mentioned at a much cheaper cost.
\end{abstract}

Keywords: Blockchain, Revenue Sharing, Security Token, Smart Contract

\section{Introduction}

Solar Photovoltaics (PVs) have played a major role in driving renewable energy sustainable development. Due to the contribution of the technology to sustainable development, the world needs to increase its rate of renewable energy adoption. The current adoption rate is not adequate to address the climate change problem. The mismatch between the current adoption rate and the needed one is even more severe in Southeast Asia (Plumer, 2018).

The low adoption rate can be partly attributed to the unbalanced concentration of solar adoption in large projects (Junlakarn and Kokchang, 2020). While Solar PVs have the potential to be a major factor in addressing the climate change, solar PV investment has thus far been concentrated in large projects. The investment concentration is caused by the high cost and the low liquidity of solar PV investment. Solar PV investment is typically done by large institution

investors (Alafita and Pearce, 2014; Junlakarn and Kokchang, 2020) because of its high cost. To improve their return on equity, large institution investors prefer to invest in large projects to leverage economies of scale. The return on equity optimization goal of large institution investors lead to an unbalanced solar PV adoption.
The concentration of solar PV investment leads to notable disadvantages. A large solar PV plant is exposed to geolocation risks. For example, a natural disaster or a localized air pollution by Particulates (PM2.5) can have devastating effects on a solar PV plant's production (Son et al., 2020). At the same time, the investment capital typically comes from large institutions which have only little connection with the community around the plant, the disconnect manifests in the apathy of the community in the area toward the governance of a solar PV plant (Joshi et al., 2019).

A more balanced solar PV investment between large and small projects leads to a more robust and sustainable solar PV adoption. A more distributed solar PV generation capacity helps reduce geolocation risks. A production reduction in one location does not affect the production other far away plants. Additionally, smaller solar PV plants provide more accessible investment opportunities for smaller investors. Since the investment is more accessible, the community living around a solar PV plant can invest and be a stakeholder of the plant. Having skin in the game, the community should participate more in the governance of the plant (Joshi et al., 2019). 
Many centralized solutions to rebalance solar PV adoption between large and small projects have failed to achieve what is necessary. The most notable form of a centralized solution is government-subsidized incentives. Such a solution has been shown to increase the solar PV overall adoption level (Crago and Chernyakhovskiy, 2017). However, it also leads to investment concentration in large projects. The kind of projects that can take a full advantage of government subsidies. More balanced solar PV development can only happen when all stakeholders, down to individual levels, are part of the solution (Joshi et al., 2019). Community involvement needs to be built-in into the development plan from the start, not post-deployment.

There are existing solutions to help balance the solar PV investment between large and small projects. The most notable solution is the aggregation of solar PV capacity into an Energy Infrastructure Fund (EIF), such as Thailand's SUPEREIF (Bualuang Fund BBL Asset Management, 2020). An EIF is a mutual fund with its portfolio consists of energy production facilities. As a mutual fund, an EIF can price its shares in an arbitrarily small denomination. The small denomination helps attract smaller investors (Priya and Joydeep, 2009). In effect, an EIF is similar to the process of crowdfunding a solar PV investment. Even though an EIF solves the community engagement problem, but it introduces a new problem in profit distribution. With many shareholders, the process of profit distribution becomes just as cumbersome as a typical mutual fund. Moreover, many checks and balances are needed to ensure the validity and the transparency of the process. The cumbersome process can incur high auditing fees. The high fees decrease the investment return of all shareholders.

Blockchain technology facilitates the process of crowdfunding. The desired features of a crowdfunding, such as small denomination, high liquidity and easy public participation, are well aligned with the strengths of the blockchain technology. Since a blockchain-based crowdfunding can be priced in a small denomination, smaller retail investors can participate in the process. A lower capital barrier for participation results in an easy public participation. An investor can diversify risks across multiple assets in different geolocations due to the small initial investment requirement. The diversification also increases the level of liquidity in all assets involved as well.

A blockchain-based platform for managing solar PV investments increases the investment accessibility. The core concept of this platform is the asset tokenization process, which transforms non-blockchain assets into security tokens. With the blockchain technology, security tokens can represent equities of a solar PV plant. An investor can invest, trade, or exchange the tokens just as one would be able to do with a share in a publicly listed company. Such platform facilitates fundraising, reduces profit distribution costs and improves a solar PV investment liquidity.

In this study, a blockchain-based platform called Decentralized Sustainable Development (DeSDev) is proposed. The platform is designed to tokenize a solar PV investment. Its notable features are small denomination tokens, high liquidity and low profit distribution costs. The three features would also encourage community participation in solar PV investment. Since its strengths improve upon the weaknesses of centralized solutions, the DeSDev platform can help increase the adoption of solar PV for sustainable development.

The rest of this study is organized as follows: Section II discusses the general process of solar PV investment and relevant stakeholders. Section III compares the solar investment process between the existing centralized solutions and the decentralized solution enabled by the use of the blockchain technology in details. Existing blockchain-based solutions and their shortcomings are also presented in this section. Section IV proposes a blockchain-based renewable energy investment management platform called Decentralized Sustainable Development (DeSDev) platform. The DesDev platform is capable of addressing the shortcomings. The implementation detail is also provided in this section. Section V provides the performance analysis of the proposed platform. Section VI concludes our analysis and provides some suggestions for future works.

\section{Solar PV Investment and its Stakeholders}

A solar investment consists of many types of stakeholders: An asset owner, an investor, a community and an electricity consumer. In this section, the role of each stakeholder type is discussed.

An asset owner starts the process by surveying for suitable installation sites. Once a suitable site is found, a supplier/general contractor to construct the project is identified. Before the construction can begin, an adequate amount of capital is sourced from a combination of sources. The rest of the process for an asset owner then proceeds in a logical manner from installation of the PV to sell the generated electricity to consumers. An asset owner takes care of the financial operations of the investment, including account receivable collection and profit distribution.

An investor is a person or a group of people who provides the capital for the project. An asset owner can also be an investor for the project (self-funding). However, in general, due to the large capital needed for a typical solar investment project, the capital is sourced from multiple investors. An investor can be broadly classified into a government, domestic capital and global capital (Fig. 1). Since an energy production facility can be considered a critical piece of a country's energy infrastructure, many countries have capital control regulations on foreign capital 
in various forms, such as a lengthy Know-Your-Customer and Anti-Money-Laundering (KYC/AML) processes or maximum investment limitation. The regulation hurdles on foreign capital reduces the efficiency of international capital movement for solar investment. The efficiency reduction results in projects that rely mostly on a government or domestic funding. It also slows down the growth rate of the solar PV installation.

Note that an asset owner does not have to own the site on which the project is built. The site can be rented from the landowner. For such cases, the landowner can be considered as an investor who contribute with a piece of land and receives the rental fee as a payment.
A consumer is an electricity user who purchases the electricity generated by the PV. The electricity payment is a source of revenue for the PV project. Lastly, the community close to the PV project receives some benefits such as clean energy usage. Additionally, the nearby community can receive a part of the project's revenue as a charitable donation. The donation helps strengthen the tie between the community and the project.

A PV project can only be realized if all the stakeholders receive what they consider as their fair shares from the project. All stakeholder types and the connection between them are shown in Fig. 2. The blockchain-based investment platform delivers services to customers is illustrated in Fig. 3.

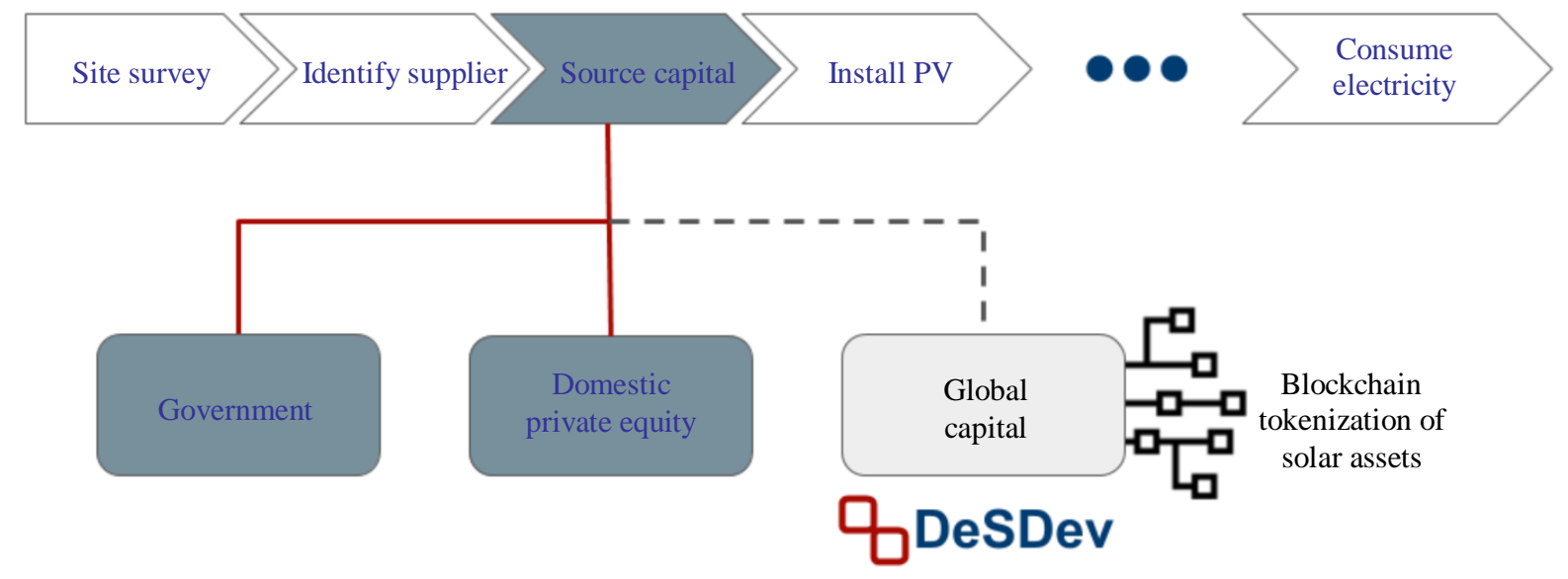

Fig. 1: Solar investment value chain



Community

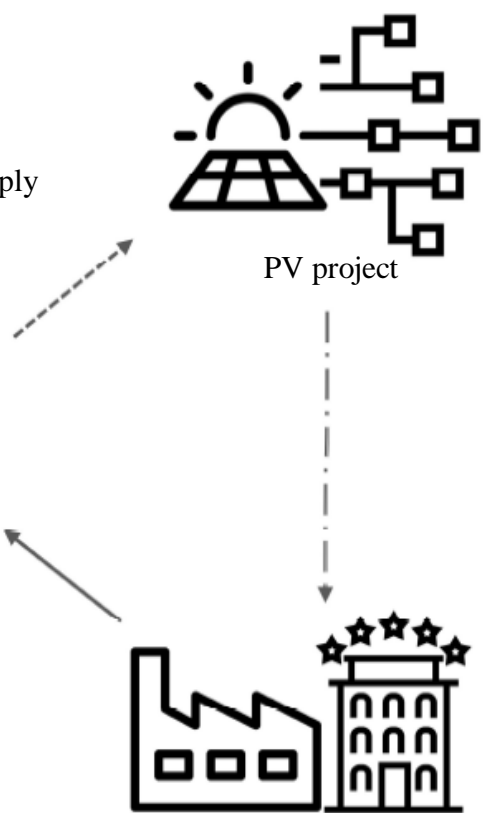

Consumer

Fig. 2: Stakeholders in a solar PV investment 


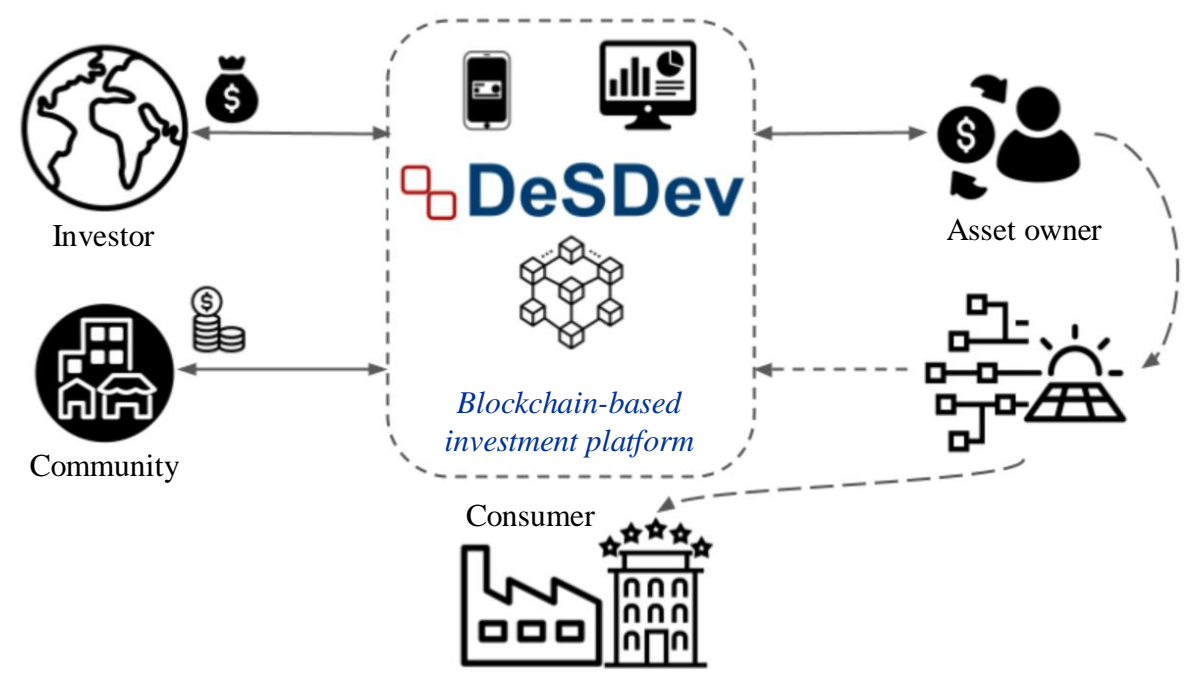

Fig. 3: Blockchain-based investment platform

\section{Solar Investment Comparison}

While many solutions exist to encourage more solar investment, all of them have shortcomings that are difficult to overcome. In this section, the general process and choices in solar investing are shown. The existing solutions and their issues are discussed.

\section{Centralized Solutions}

Without a decentralized blockchain-based solution, the current investment schemes for solar PV are as follows: A sole ownership, a Power Purchase Agreement (PPA) and an EIF. A sole ownership is when an asset owner has enough capital to solely invest in a solar PV project. Only small amount of projects are invested in this way due to the large capital involved in the process. A sole owner bears all the risks and benefits of the investment. A sole ownership carries the most investment risk on account of the exposure to geolocation risks and the potential production reduction.

A PPA allows an asset owner to receive a steady income from the generated electricity by getting into a power purchasing contract with electricity consumers. Typically, an asset owner would rent a physical space for the installation of solar PVs. In most cases, the consumers are also the owners of the physical space. A PPA reduces investment risks of the asset owner. The contract generates a steady stream of income throughout the lifetime of the contract. However, a PPA does not address the exposure to geolocation risks. Diversification of solar PV capacity is difficult to do in this investment model.

An EIF can diversify the solar PV capacity over different geolocation areas. It is also easier to invest in a share of an EIF that can be denominated at low price. However, an EIF introduces other problems not existing in other centralized solutions. Some of the problems are the difficulty of a real-time revenue tracking, the high fees associated with checking the investment for validity and transparency and the lengthy process in profit distribution.

For the centralized solutions, the generated revenue is tracked in many steps. Energy generation data is recorded by a smart meter. The records are stored in a centralized database that requires backup and maintenance. The transactions involved then have to be verified before the profit can be distributed to investors. The long verification leads to slow profit distribution. Moreover, the process is opaque and difficult to inspect for transparency. The entire process is shown on the right hand side of Fig. 4.

\section{Blockchain-based Solutions}

Rather than relying on a centralized database or a manual verification of transactions, a blockchain-based solution can be implemented.

The blockchain technology was first proposed by (Nakamoto, 2019) a pseudonym of yet-anonymous person(s). The technology creatively combines cryptography with a decentralized database, which allows a blockchain to be decentralized, immutable and transparent at the same time. Many studies have analyzed the technology in detail (Li et al., 2020; Upadhyay, 2020). Interested readers are encouraged to follow the references. For the current purposes, certain features of the blockchain technology that are able to solve the problems found in centralized solutions are pointed out.

A token representing a non-blockchain asset allows any assets to be priced in arbitrarily small denominations. Since any number of tokens can be created on a blockchain to represent an asset with a finite monetary value, a token can have small denomination. The small denomination enables participation from small investors, which helps rebalance the distribution of solar PV capacity between large and small projects. 


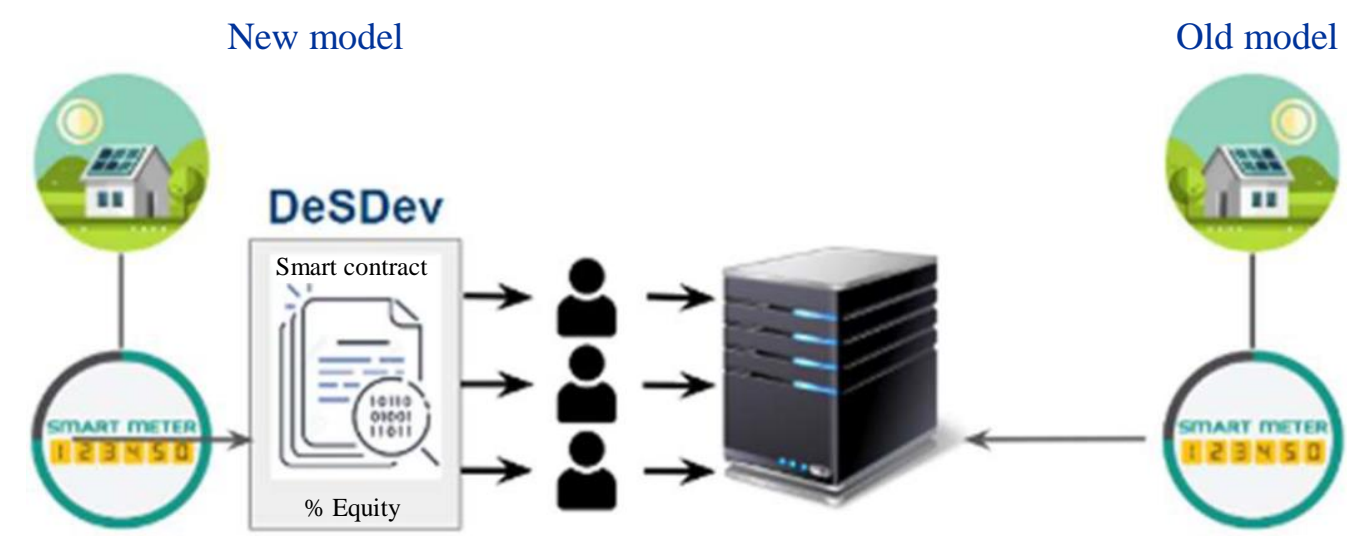

Fig. 4: Comparing centralized solutions with a blockchain solution

The same set of tokens can represent various assets in different geolocation. Therefore, an investment in a token can expose an investor to multiple assets at the same time. The variety in risk exposure in different geolocation allows an investor to easily diversify investment risks.

A blockchain-based solution can distribute profit to shareholders cheaply. In contrary to the traditional EIF structure, which requires accountants and auditors to ensure the validity and transparency of transactions, a blockchain has both features built right into the protocol (Karode et al., 2020; Li et al., 2020). In other words, it is nearly impossible to have a transaction on a blockchain that is not valid or not transparent. Some exceptions do exist ( $\mathrm{Li}$ et al., 2020). However, for the purpose of managing solar PV investment, the exceptions can be handled easily by using a permissioned blockchain.

Most importantly, the profit distribution process can be handled transparently by a smart contract. A smart contract was first implemented on the Ethereum blockchain (Wood, 2014). A smart contract works the same way as a typical paper legal contract. The difference is how a contract is enforced. For a paper contract, a breach of contract requires an arbitration by a judicial system, which can be costly and time consuming. A smart contract enforces the content of the contract strictly and predictably, which leads to higher confident and transparency for financial transactions.

Unlike the EIF solution, a blockchain solution can track revenue generation in real time. Since all data regarding energy production and revenue distribution are represented digitally on a blockchain, the capital table and profit distribution process can be tracked in real time. The real-time tracking feature can only be realized in a cost-efficient manner with a blockchain solution.

Blockchain-based solutions have already been implemented successfully in various markets due to the advantages mentioned above. For example, The Aspen Digital Security Token represents an equity in St. Regis
Aspen Resort, Switzerland (Aspen, 2020). A token holder is entitled to share the resort's revenue, but not its ownership. Thus, asset tokenization also unbundles the rights to revenue from the rights of ownership as well. Another example is (RealT Inc, 2020). The company specializes in tokenizing real estates in the US. Interested customers can buy tokens representing various real estates. The rental profit from the real estates is distributed cheaply and frequently to investors from all over the world.

There is only one existing blockchain-based solution for solar PV investment management. The Digital Currency initiative has proposed the OpenSolar project. OpenSolar first issues a municipal bond, which is offered to general investors. The money raised from the municipal bond is used to develop various solar PV projects. The capital table management and revenue tracking are done on a blockchain. OpenSolar uses the Ethereum blockchain to handle all the transactions. The revenue is then converted to fiat money and paid back to the investors in the form of the interest of the municipal bond.

While the OpenSolar project solves many problems found in centralized solutions and also implements a blockchain-based solution, many obstacles still hinder mass adoption of solar PV blockchain solution. For example, the use of municipal bonds excludes the influx of global investment. Only local investors are eligible to participate in municipal bond investments in both cases. Additionally, the process of converting from crypto tokens into fiat still faces the same high fees found in the EIF solution (OpenSolar, 2020). OpenSolar's reliance on some traditional finance structures and practices, such as bonds and account auditors, limits the project's scalability. The costs associated with expanding in OpenSolar grows supralinearly with the installed capacity. Therefore, OpenSolar provides only a partial solution in accelerating solar PV adoption. 


\section{The Decentralized Sustainable Development (DeSDev) Platform}

The shortcomings of the existing blockchain-solution identified in the prior section can be addressed using a smart contract implementation presenting within the blockchain technology. In this section, a blockchainbased platform called Decentralized Sustainable Development (DeSDev) is proposed. How DeSDev addresses the shortcomings is discussed. Implementation specification of DeSDev is presented. The potential for DeSDev to scale up sublinearly with the total system capacity is shown in Fig. 5. DeSDev consists of the three main layers.

\section{Blockchain Layer}

DeSDev is implemented on the Ethereum blockchain as a smart contract. A smart contract is a set of functions and properties that are programmed onto a blockchain. The smart contract executes and/or controls transactions automatically. DeSDev's smart contract has both security tokens and a custom function control. An important part of the smart contract involving profit distribution is shown in Algorithm 1.

All transactions executed and processed by DeSDev's smart contract are recorded on the Ethereum blockchain. The investors can verify all transactions either through the DeSDev user interface or any popular explorers for the Ethereum blockchain, such as Etherscan (Fig. 6).

The smart contract is implemented using Solidity language (Dannen, 2017). The smart contract represents all assets in the system as tokens conforming to
Ethereum Request for Comment 20 (ERC-20). ERC-20 standards are chosen since all ERC-20 tokens can interact seamlessly with one another on the Ethereum blockchain (Buterin and Vogelsteller, 2015). The seamless integration between all ERC-20 tokens also accommodates global capital investment through various stablecoins existing on the Ethereum blockchain (Sidorenko, 2019). Additionally, DeSDev tokens can be listed on many exchanges, such as 0x exchange (Warren, 2020). An exchange listing improves the liquidity of DeSDev tokens as well.

\section{System and UI/UX Layer}

The system can be divided into the frontend and the backend infrastructures. Bootstrap, React and Web3JS are used to develop DeSDev's frontend. NodeJS and Firebase are used for the backend with the help of CircleCI and Docker for server infrastructures and operations. Not all data will be written onto the Ethereum blockchain. While storing all data on the blockchain is more transparent, however each data I/O process incurs a small fee (called gas in Ethereum's parlance). DeSDev stores non-financial data in a centralized database. Instead, DeSDev opts for storing some non-financial data (e.g., user profiles) in Firebase. Partial data storage in Firebase helps reduce the operating costs of DeSDev.

\section{User Layer}

This layer consists of humans who interact with DeSDev, both as administrators, users, or even the general population.

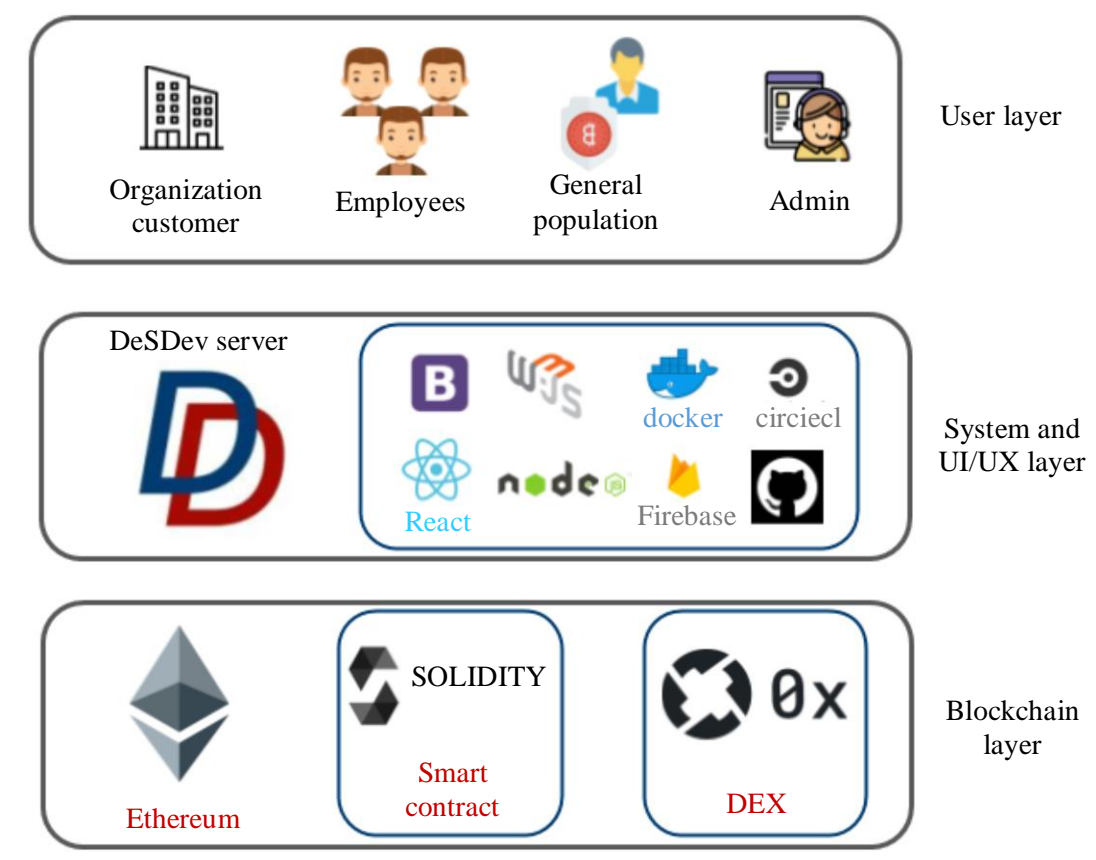

Fig. 5: The platform layers 


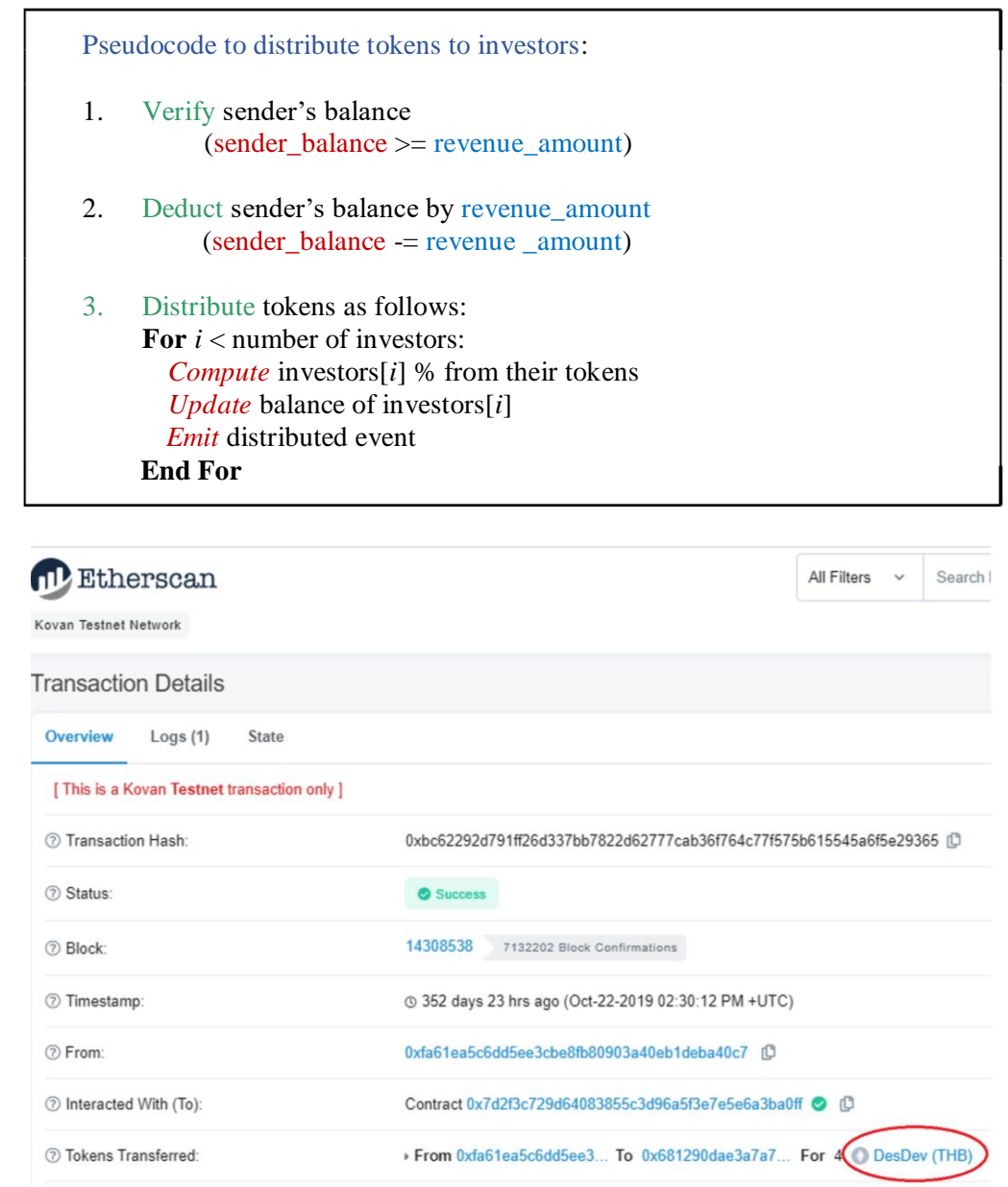

Fig. 6: Transaction in Ethers can explorer

\section{Performance Analysis}

The DeSDev platform has to handle financial transactions transparently, correctly and cheaply. The transparency requirement is natively supported by the Ethereum blockchain, which records and publicizes all transactions.

DeSDev is bench-tested on a simulated data to establish the correctness of transactions handled by the DeSDev platform. A known capital table of multiple investors is varied over time to show that DeSDev can track the changing equity shares. The profit distribution performed by DeSDev is then compared to a known result. The gas use analysis is done over changing use conditions, from small to large scale operations to establish that DeSDev is cheaper than their centralized solution counterparts.

\section{Photovoltaic Power Generation Simulation}

To investigate the correctness of the DeSDev platform using a set of data as close as possible to the real-world environment, a 20-year revenue from electricity sales was simulated. The period of 20 years was chosen to coincide with the typical warranty length of solar PV. A 1-MWp nominal power crystalline silicon Photovoltaic (PV) was used as a case study. The chosen nominal power represents a typical commercial scale PV. The specifications of the case study PV can be found in Table 1. The Photovoltaic Geographical Information System (PVGIS) was used to simulate electricity generation of the case study for 20 years (European Commission Joint Research Centre, 2019).

Using the specifications of the case study, the PVGIS estimates the solar radiation intensity from satellite data. The solar radiation intensity can be used to calculate the amount of radiation received by the PV. The amount of radiation received is then converted into the PV power output for each hour of a typical year. Additionally, the PVGIS calculates the variation in the power output using an ensemble of past weather data, its effects on the temperature of the PV and the sunset and sunrise hours. The resolution of the PV output variation is monthly. 
Table 1: Specifications of the case study photovoltaic

\begin{tabular}{ll}
\hline Location & Phuket, Thailand \\
\hline Latitude & 7.890 \\
Longitude & 98.399 \\
Elevation (m) & 18 \\
Slope (deg.) & 0 \\
Azimuth (deg.) & 0 \\
Nominal power & $1 \mathrm{MWp}$ \\
System losses (\%) & 4 \\
\hline
\end{tabular}

From the hourly data simulated by the PVGIS, a Monte Carlo method was used to simulate the hourly output of the solar PV over 20 years. Each data point is a random sampling of a normal distribution described by the Equation 1:

$$
E_{t, d, m} \sim N\left(\mu_{t, m}, \sigma_{m}^{2}\right),
$$

where, $E_{t, d, m}$ is the energy output of the PV at hour of the day $t$, on day of the year $d$ and month $m, \mu_{t, m}$ is the mean energy output at $(t, m)$ and $\sigma_{m}^{2}$ is the variance of the output in month $m$.

The Monte Carlo simulation allows the performance of the DeSDev platform to be tested with a dataset of electricity sales revenue consisting of hourly, daily and monthly variations as close to the real-world environment as possible. The variation of energy generation from the average generation is shown in Fig. 7.

\section{Changing Capital Table and Profit Distribution}

The simulated electricity generation data is fed to DeSDev's smart contract. In the smart contract, four dummy investors with various equity shares are represented by four Ethereum wallets, which are public keys on the Ethereum blockchain. The smart contract searches for the capital table of the investors. The profit from the electricity generation is then distributed to each investor according to the investors' equity shares.

Since the capital table is known ahead of time, the correct profit distribution ratio can be analytically solved. The result distribution from the smart contract agrees with the expected analytical result. The DeSDev platform can distribute the profit correctly, even under a constantly changing capital table. The distribution ratio is shown in Fig. 8.

\section{Transaction Speed}

Another advantage of the DeSDev platform is the transaction speed. The simulated solar PV generation data is used as an input to estimate the transaction speed of an average transaction.
Total transaction time is used to estimate a typical transaction speed of the DeSDev platform. Total transaction time consists of hash time and block time. All measurements include data request and response time. Hash time is the duration from sending a signed transaction until the Ethereum blockchain responds with a transaction hash. Having a hash means that the transaction is broadcasted to the Ethereum network, but it is not finalized. Block time is the duration from sending a transaction until it is recorded in a block on the Ethereum blockchain. Hash time is a subset of block time.

There are two steps involved in a profit distribution. First, the profit available for distribution is taken from the smart contract. Then the profit is delivered to the investors according to the capital table at the moment of distribution. Both steps take on average $1.9 \mathrm{sec}$ for hash time (Fig. 9). The block time is longer with an average of 27.6 seconds (Fig. 10). Therefore, the DeSDev platform can distribute the profit frequently and nearly in real-time.

\section{Transaction Costs}

The DeSDev platform needs to scale better than existing centralized solutions to accommodate the needed exponential growth of Solar PV adoption. The cost to perform the needed transaction to be less in DeSDev than through traditional banking systems.

In an EIF, transactions are done through bank money transfer services, such as the SWIFT system. The profit distribution cost through such a system is shown in the Equation (2):

Total bank costs $=$ transfer fee $\times$ no.of users.

Total DeSDev costs $=$ Gas used $\times$

Gas price $\times$ ETH price $\times$ no. of users

The minimum transfer fee for a bank transaction internationally is 12.61 USD. A typical transfer fee could be much greater than this amount. The lowest fee amount is used in comparing DeSDev to centralized solutions.

On the contrary, DeSDev distributes the profit through a smart contract.

The amount of Gas used is estimated by submitting multiple transactions through the smart contract using the simulated solar PV generation data. The average amount of gas used is 350,690 units (Fig. 11). Gas Price converts the units of gas used into Ethereum amount. The average price at the time of writing is used. The average price is $47 \mathrm{GWei}\left(1 \mathrm{ETH}=10^{9} \mathrm{GWei}\right)$. ETH price averages to 272.12 USD at the time of writing. The total DeSDev costs is calculated by the Equation (3). 
The total transaction costs on DeSDev is cheaper than the total costs in centralized solutions. The comparison of the total costs at various number of investors is shown in Fig. 12. Since the growth in transaction costs of the DeSDev platform rises slower with an increasing number of investors, the DeSDev platform scales to accommodate the needed exponential growth of solar PV adoption better. Moreover, since DeSDev's smart contract is geolocation agnostic, the DeSDev platform is more suitable for the modern financial world with globallevel flow of investment capital.

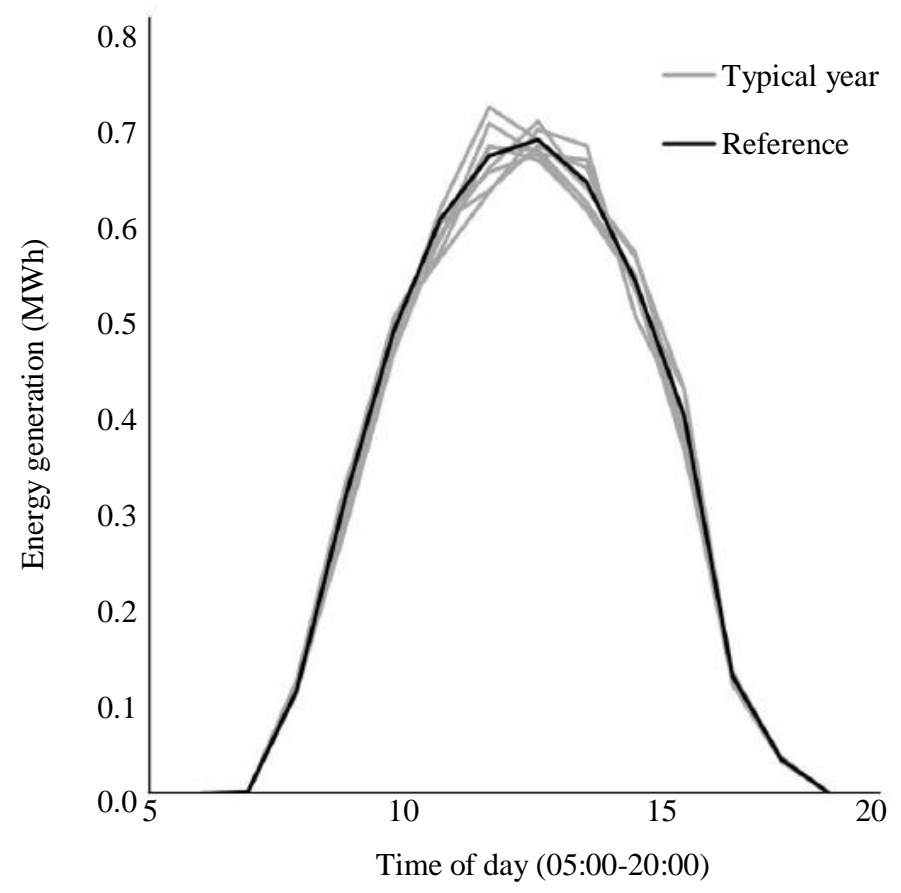

Fig. 7: The variation of energy generation as compared to the reference (mean) year. Hourly resolution in a day shown

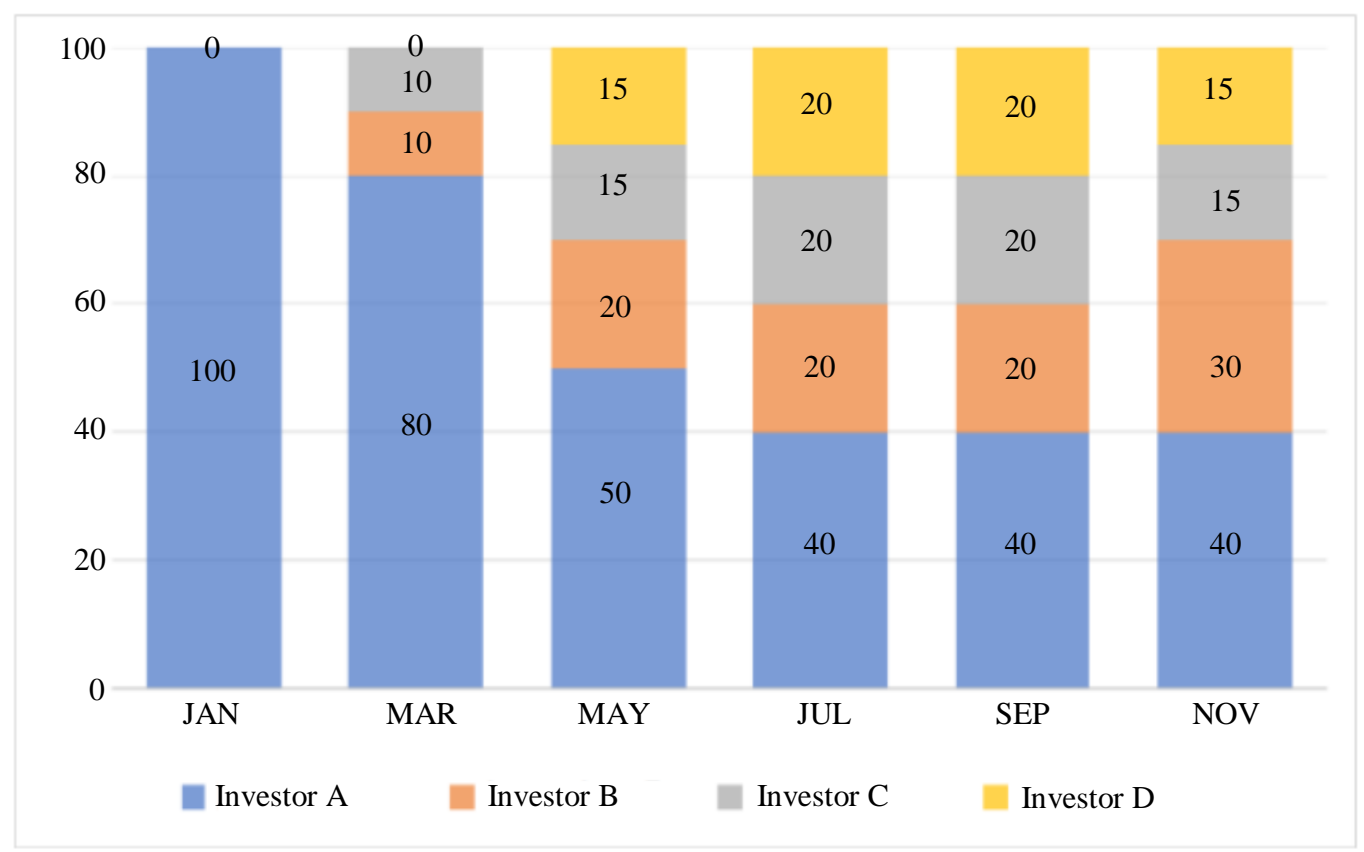

Fig. 8: The profit distribution ratio under a changing capital table 


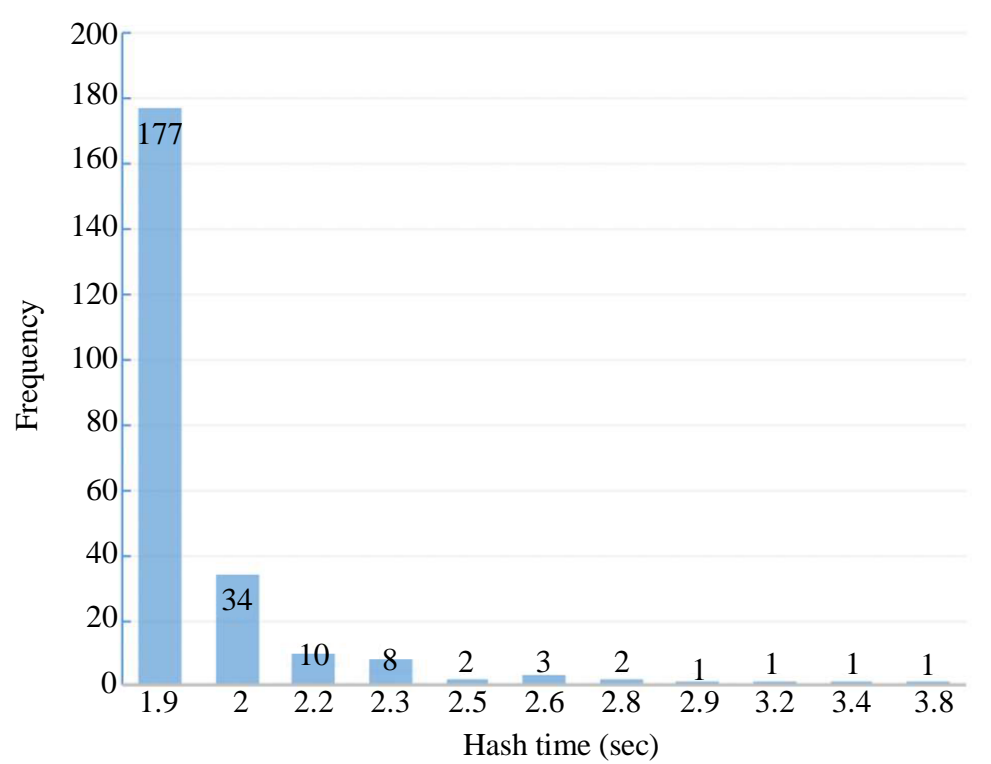

Fig. 9: Hash time histogram to deposit to the smart contract and distribute revenue to all investors

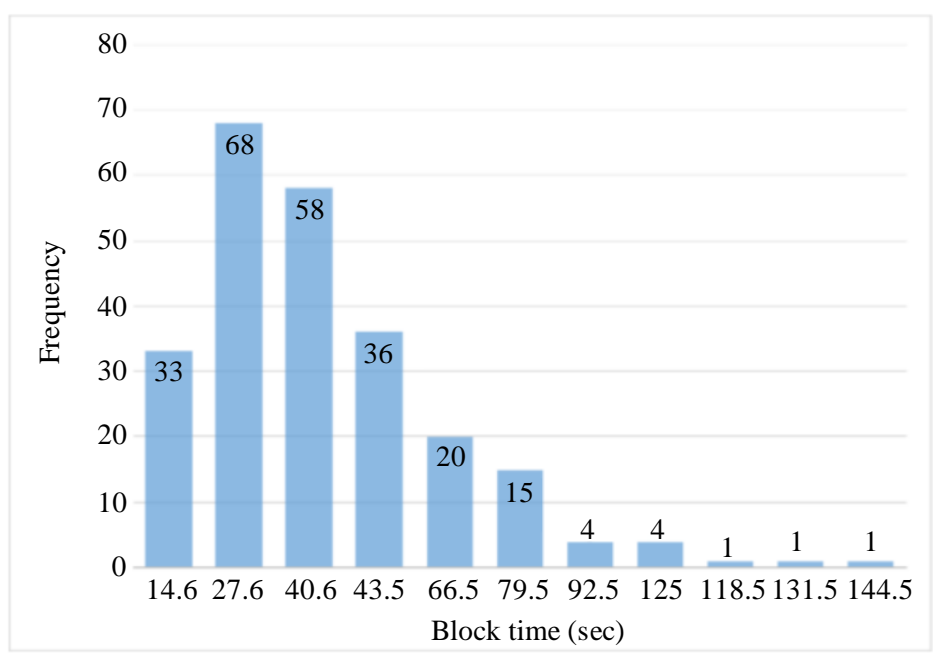

Fig. 10: Block time histogram to deposit to the smart contract and distribute revenue to all investors

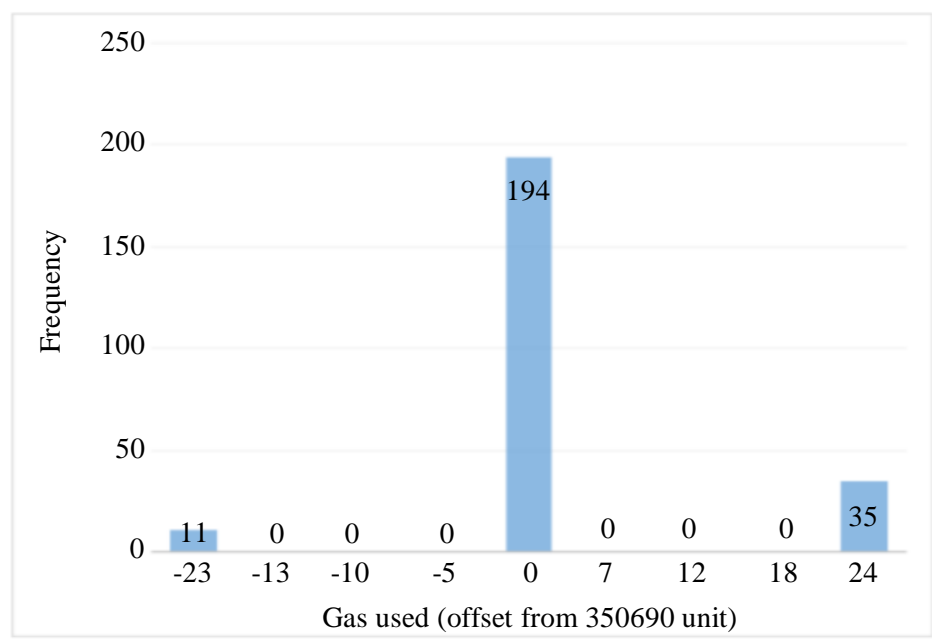

Fig. 11: Gas used histogram to deposit to the smart contract and distribute revenue to all investors 


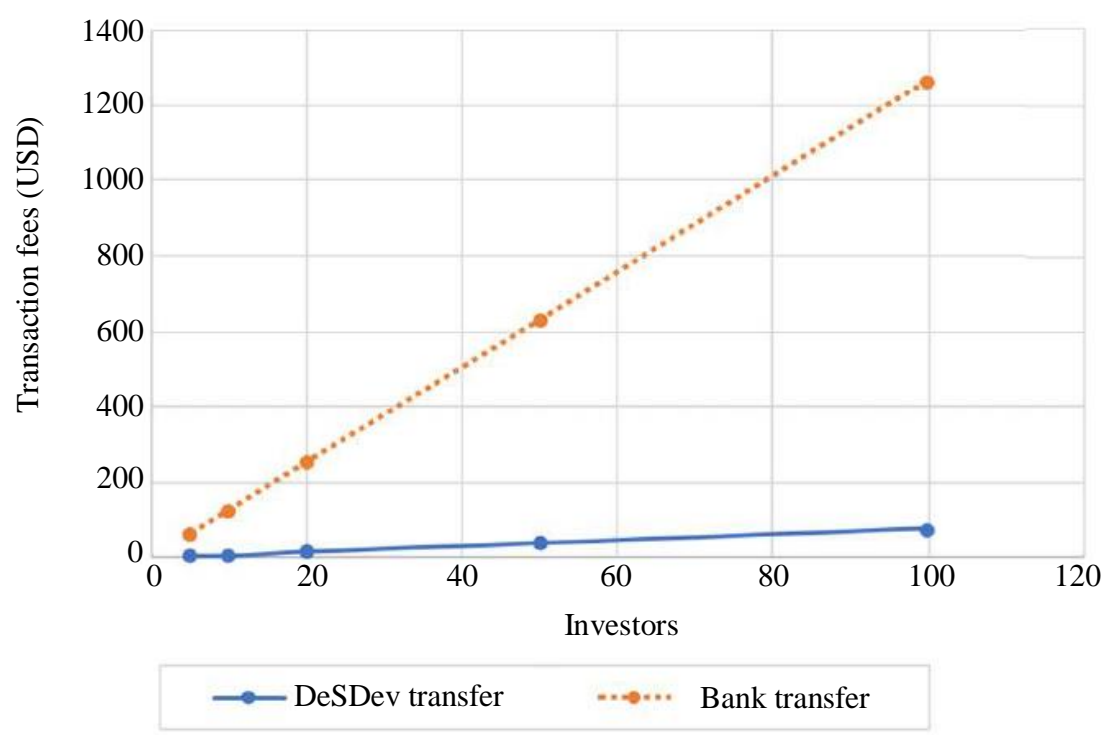

Fig. 12: Transaction fee comparison

\section{Conclusion and Future Works}

The DeSDev platform was tested using a simulation data. In order to stress test the platform in more realistic scenarios, a benchtop experiment can be used to generate real-time noise-included power generation data from a small-scale solar PV. The performance of the platform under a more realistic scenario can then be established. Additionally, the fluctuating Ethereum price creates a large uncertainty in total transaction costs. The uncertainty can be quantified and confidence intervals for total transaction costs can be established. Lastly, the platform could incorporate some forms of Ethereum price hedging by exchanging between Ethereum and other cryptocurrencies (e.g., stablecoins). The hedge could give the platform a method to reduce its exposure to Ethereum's fluctuating price.

In addition to the technical aspects of DeSDev, regulatory aspects have to be considered as well. For example, the identity verification (commonly known as the KYC/AML process) has to be compared between centralized solutions and DeSDev. The willingness of national governments to accept trans-national transactions and taxation.

Nevertheless, we have presented a renewable energy investment management platform based on blockchain technology, called DeSDev. The research contribution is not only to propose the platform but also to present a business model applied to this platform that offered many advantages with the performance analysis. The platform has several advantages in terms of transparency, flexibility and cost over existing solutions. Moreover, all transactions are carried out near real-time. The technical implementation of DeSDev on the Ethereum blockchain was discussed. The performance and costs of the platform were analyzed and compared to existing solutions. The DeSDev platform offers a blockchain-based solution that is more decentralized than other existing blockchain-based solutions. At the same time, it is more transparent, flexible and cheaper than centralized solutions. DeSDev has the potential to exponentially increase the adoption of solar PV due to its strong features.

\section{Acknowledgment}

The authors acknowledge the support of College of Computing, Prince of Songkla University, under the BLOCK research team.

\section{Funding Information}

This research was supported by Thailand Fundamental Research 2020-2021, and T. Arpornthip was funded by Thailand Research Fund, grant number MRG6280201.

\section{Author's Contributions}

Warodom Werapun: Contributed substantially in the topic and analyzed the literature along with proposing and implementing the methods, conducting the simulation experiments and perform all other research works.

Tanwa Arpornthip: Conceptualized the topic direction, provided research materials and resources. Undertaked checking and validations of all key information of the paper.

Esther Sangiamkul: Created paper presentation graphics, checked and validated key results of the paper. 
Rattana Wetprasit: Contributed to the manuscript review, discussed the research and provided encouraging suggestions and recommendations.

Tanakorn Karode: Worked in preparing the smart contract and gas calculation for experimental results and discussed the content of the paper intensively.

\section{Ethics}

This research manuscript is original and has not been published previously and not under consideration for publication elsewhere, in whole or in part. The corresponding author confirms that all of other authors have read and approved the manuscript and there were no ethical issues involved.

\section{References}

Alafita, T., \& Pearce, J. M. (2014). Securitization of residential solar photovoltaic assets: Costs, risks and uncertainty. Energy Policy, 67, 488-498.

Aspen. (2020). Aspen, Colorado Luxury Resort | The St. Regis Aspen Resort. https://www.marriott.com/hotels/travel/asexr-the-stregis-aspen-resort/

Bualuang Fund BBL Asset Management. (2020). SUPEREIF annual report 2019. Bualuang Fund BBL Asset Management. http://supereif.listedcompany.com/misc/ar/20200413 -supereif-ar2019-en.pdf

Buterin, V., \& Vogelsteller, F. (2015). Erc20 token standard. https://theethereum. wiki/w/index. php/ERC20 Token Standard.

Crago, C. L., \& Chernyakhovskiy, I. (2017). Are policy incentives for solar power effective? Evidence from residential installations in the Northeast. Journal of Environmental Economics and Management, 81, 132-151.

Dannen, C. (2017). Introducing Ethereum and solidity (Vol. 1). Berkeley: Apress.

European Commission Joint Research Centre. (2019). Photovoltaic Geographical Information System (PVGIS).

https://re.jrc.ec.europa.eu/pvg_tools/en/\#PVP

Joshi, L., Choudhary, D., Kumar, P., Venkateswaran, J., \& Solanki, C. S. (2019). Does involvement of local community ensure sustained energy access? A critical review of a solar PV technology intervention in rural India. World Development, 122, 272-281.
Junlakarn, S., \& Kokchang, P. (2020). Comparative customer economics on different financial options in support the adoption of residential rooftop photovoltaic in Thailand. E\&ES, 463(1), 012145.

Karode, T., Werapun, W., \& Arpornthip, T. (2020). Blockchain-based Global Travel Review Framework. International Journal of Advanced Computer Science and Applications, 11(8). https://doi.org/10.14569/IJACSA.2020.0110813

Li, X., Jiang, P., Chen, T., Luo, X., \& Wen, Q. (2020). A survey on the security of blockchain systems. Future Generation Computer Systems, 107, 841-853.

Nakamoto, S. (2019). Bitcoin: A peer-to-peer electronic cash system. Manubot.

OpenSolar. (2020). MIT Digital Currency Initiative. https://dci.mit.edu/opensolar

Plumer, B. (2018, November 12). Clean Energy Is Surging, but Not Fast Enough to Solve Global Warming. The New York Times. https://www.nytimes.com/2018/11/12/climate/global -energy-forecast.html

Priya, R., \& Joydeep, S. (2009). The Denomination Effect. Journal of Consumer Research, 36(4), 701-13.

RealT Inc. (2020). Fractional and frictionless real estate investing. RealT, Inc. https://realt.co/

Sidorenko, E. L. (2019, April). Stablecoin as a new financial instrument. In International Scientific Conference "Digital Transformation of the Economy: Challenges, Trends, New Opportunities" (pp. 630-638). Springer, Cham.

Son, J., Jeong, S., Park, H., \& Park, C. E. (2020). The effect of particulate matter on solar photovoltaic power generation over the Republic of Korea. Environmental Research Letters, 15(8), 084004.

Upadhyay, N. (2020). Demystifying blockchain: A critical analysis of challenges, applications and opportunities. International Journal of Information Management, 54, 102120.

Warren, W. (2020). 0x: Powering the decentralized exchange of tokens on Ethereum. https://0x.org/

Wood, G. (2014). Ethereum: A secure decentralised generalised transaction ledger. Ethereum project yellow paper, 151(2014), 1-32. 